ESAIM: PROCEEDINGS, April 2009, Vol. 26, p. 135-149

H. Ammari, Editor

\title{
IMAGERIE THERMIQUE, LASER ET MODÉLISATION EN BIOPHOTONIQUE
}

\author{
G. BERGINC ${ }^{1}$
}

\begin{abstract}
.
Résumé. Cet article introduit deux concepts en imagerie médicale. D'une part, nous aborderons l'utilisation de l'imagerie thermique dans l'infrarouge lointain ou thermographie en biophotonique. Ainsi grâce aux progrès récents des capteurs dans la bande infrarouge, une cartographie thermique précise des régions d'intérêt du corps humain peut être déterminée. D'autre part, nous introduirons l'imagerie laser ou plus généralement l'imagerie active par opposition à l'imagerie thermique qui n'utilise aucune source de lumière supplémentaire. Ce type d'imagerie active ou laser utilise principalement l'intensité diffusée ou rétro diffusée par le tissu sous l'éclairement d'une source spectralement étendue ou laser. Cette approche non invasive peut être multispectrale polarisée et combinée à des modèles numériques physiologiques. Multispectrale, elle nécessite plusieurs sources d'éclairement dans la bande visible et proche infrarouge, cette notion de multispectralité de la source d'éclairement permet d'optimiser la profondeur de pénétration de l'onde dans le tissu considéré. Polarimétrique, elle permet de discriminer la dépolarisation de l'onde électromagnétique au cours de son parcours dans les tissus et de sa diffusion sur les interfaces du tissu. Nous présenterons l'ensemble des imageries optiques tomographiques. Nous développerons aussi les modèles électromagnétiques permettant de simuler la diffusion d'une onde par une structure multicouche comprenant un volume aléatoire et des interfaces rugueuses aléatoires. Cette structure ainsi décrite modélise la peau. Au cours de cet article, nous nous attacherons plus particulièrement à l'analyse de la peau.
\end{abstract}

\section{INTRODUCTION}

Nous pouvons définir la biophotonique comme l'utilisation du spectre ultraviolet, visible, proche infrarouge et infrarouge pour l'analyse ou la modification d'objets biologiques. La biophotonique peut être considérée comme l'une des applications les plus porteuses des sciences et technologie de la photonique et de l'optronique. Son développement semble être très prometteur sur le plan économique, il participe aux progrès de la santé par exemple : nouveaux types d'analyse et de soins, facilité et fiabilité des diagnostics, progrès de la cosmétologie et maîtrise raisonnée de l'environnement. La biophotonique constitue un enjeu sociétal considérable, elle va contribuer fortement à l'amélioration de la santé humaine et de l'environnement. Dans le domaine de la lutte contre le cancer, de nouvelles technologies biophotoniques vont permettre dans les prochaines années un dépistage plus précoce et plus fiable. La biophotonique constitue un enjeu technologique fondamental. Les technologies qu'elle représente servent de nombreuses applications et sont l'élément différenciant de systèmes d'analyse plus complexes. Le croisement des savoir-faire des domaines photoniques et des sciences du vivant constitue un véritable challenge des années futures tant au point de vue de la formation que de la recherche fondamentale et appliquée et de l'industrie. L'enjeu économique est d'importance : en 2005, la biophotonique représentait déjà une dizaine de milliards d'euros. La biophotonique constituera de nouvelles richesses sur

1 Thales Optronique 2, avenue Gay-Lussac CS 90502, 78995 Elancourt Cedex, France; E-mail: gerard.berginc@fr.thalesgroup.com

(C) EDP Sciences, SMAI 2009 
un plan scientifique et en termes de création d'entreprises et d'emplois. La recherche en biophotonique est actuellement en pleine croissance dans les laboratoires publics et industriels. Les avantages de ces techniques photoniques non invasives sont nombreux notamment dans le cadre de l'aide au diagnostic des cancers de la peau. Elles utilisent des radiations non-ionisantes et peuvent révéler un contraste chimique important, elles apportent une information physiologique fiable. En effet, les contrastes obtenus par imagerie optique active sont liés aux interactions entre une onde électromagnétique dans les bandes visible ou proche infrarouge et les différents tissus organiques. Ainsi, dans le proche infrarouge, les interactions observées sont dues aux phénomènes d'absorption et de diffusion des tissus. Pour l'imagerie thermique dans l'infrarouge lointain, le capteur reçoit d'une manière non nocive le rayonnement thermique du corps humain. Au cours de ce papier, nous allons dans un premier temps introduire les progrès de la thermographie grâce aux sensibilités accrues des détecteurs dans l'infrarouge lointain (bande infrarouge couvrant le spectre de 8 à 12 micronmètre). Dans un second temps, nous allons présenter les différents types d'imagerie optique permettant de réaliser une tomographie des tissus. Nous allons en outre introduire un type d'imagerie biophotonique active non invasive de 2ème génération permettant le diagnostic et le suivi longitudinal des désordres cutanés. Le système repose sur la combinaison d'une imagerie active c'est-à-dire utilisant l'émission d'une source de lumière incohérente ou cohérente dans la bande visible ou proche infrarouge qui sera analysée par le bloc détection du système. Ce système d'imagerie peut être multispectral c'est-à-dire utilisant plusieurs bandes ou plusieurs longueurs d'onde d'émission et polarimétrique c'est-à-dire utilisant la polarisation de la lumière qui permet d'apporter des informations structurelles et fonctionnelles nouvelles. Dans une troisième partie, nous aborderons la propagation de la lumière polarisée dans un tissu ou, d'une manière plus générale, dans un milieu diffusant comprenant des interfaces rugueuses aléatoires. Il est nécessaire de connaitre les paramètres optiques caractérisant les tissus puisqu'ils peuvent contenir l information utile au diagnostic optique. Il est alors fondamental de tenir compte du problème posé par la diffusion multiple des ondes électromagnétiques polarisées dans les tissus et au passage des interfaces rugueuses aléatoires.

\section{IMAGERIE THERMIQUE}

La température du corps humain est d'environ $36.85^{\circ} \mathrm{C}$ ou $310 \mathrm{~K}$, ainsi le rayonnement produit par le corps humain se situe dans l'infrarouge lointain comme nous le voyons sur la figure 1 où le rayonnement en fonction de la température est donné par la loi du corps noir. Ainsi lorsqu'on désire effectuer en milieu hospitalier une thermographie cutanée, on mesure la température équivalente limite en surface et non la température interne. Grâce à ce type de mesure et avec un système d'acquisition calibré, une cartographie thermique précise des régions du corps humain peut être effectuée. Cette méthode consiste à mesurer à distance le rayonnement infrarouge émis par la surface dont on veut connaître la température. Ce rayonnement dépend de la température superficielle, mais aussi de l'état de surface, de sa nature et de son émissivité propre dépendant de l'angle d'émission. Bien que l'évaluation de la température de surface par la thermographie infrarouge introduise une erreur systématique lors de la mesure, cette méthode de mesure permet de dresser une carte thermique très étendue sans nécessiter de contact. Il est alors possible de détecter la présence d'une tumeur sous-cutanée, un cancer du sein par exemple, de déceler la reconstitution du système sanguin autour d'une fracture en voie de consolidation. En utilisant le système circulatoire du corps humain et l'imagerie thermique en infrarouge lointain, il est possible d'aider au diagnostic de maladies dégénératives, lors d'une mauvaise circulation sanguine, il apparaît un gradient thermique par contraste entre des organes sains et des organes pathologiques. Nous pouvons citer par exemple des maladies inflammatoires qui touchent les articulations comme l'arthrite.

Il faut remarquer que la thermographie cutanée présente un intérêt considérable pour le patient puisqu'il s'agit d'une technique non traumatisante contrairement aux méthodes par scanner ou par scintigraphie qui nécessitent l'injection d'un produit radioactif permettant de rendre visible le réseau de circulation sanguine. Parmi les techniques non-irradiantes, nous pouvons citer l'IRM qui reste une technique chère, peu disponible mais disposant d'une excellente sensibilité, l'écographie qui est une technique peu chère mais à faible sensibilité et fortement dépendant de l'opérateur. D'autres techniques non-irradiantes existent ainsi l'impédancemétrie, l'absorptiométrie optique, et la radiométrie micro-onde. Celle-ci est liée à la détection des ondes hyperfréquences 


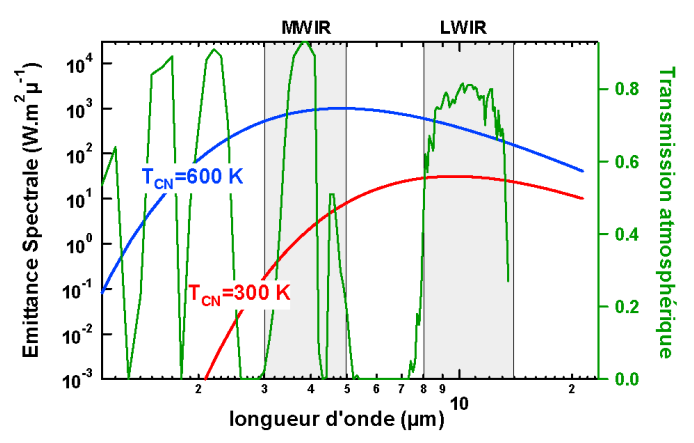

Figure 1. Loi du corps noir et fenêtres de transmission atmosphérique.

d'origine thermique rayonnées par la matière. Malheureusement, cette méthode présente l'inconvénient de ne pas se limiter à une zone superficielle et de traiter des signaux de faible amplitude et fortement bruités, ce qui rend difficile l'extraction de la température superficielle.

La réalisation d'une caméra permettant la thermographie cutanée est liée à la performance dans la bande infrarouge (8-12 micronmètre) du détecteur constituant le cœeur de celle-ci. Les détecteurs peuvent être ainsi classés en deux catégories : - les détecteurs thermiques (thermocouples, microbolomètres ...), ils sont nonrefroidis et possèdent l'avantage de travailler à température ambiante. Ils sont à faible coût mais possèdent l'inconvénient d'avoir de faibles performances, - les détecteurs quantiques. Pour la bande 8-12 micronmètre, deux types de structures semi-conductrices peuvent être utilisés, le MCT en barrettes ou petites matrices 128x128, le QWIP (Quantum Well Infrared Photodetector : photodétecteur infrarouge à puits quantiques à base d'AsGA) en grandes matrices 640x512. Les détecteurs quantiques doivent être refroidis et nécessitent un système cryogénique. Ce qui augmente fortement leur coût, par contre eux possèdent une grande sensibilité.

Les Qqn sont bien adaptés pour les applications d'imagerie nécessitant une grande résolution et une grande sensibilité, ils permettent en outre une cadence de plus de cinquante images par seconde. Ce qui correspond aux spécifications de l'imagerie médicale. Le QWIP est un détecteur quantique comme nous l'avons vu, l'absorption du rayonnement se fait par l'intermédiaire d'une transition électronique. L'absorption est résonante, le détecteur absorbe donc dans une bande étroite de longueurs d'ondes, l'absorption est caractérisée par un pic (figure 2) donné par la position de son maximum et par sa largeur à mi-hauteur. La figure 2 donne la réponse spectrale en Ampères par Watt, cette grandeur permet de connaître la plage spectrale de sensibilité du détecteur, elle est définie comme le rapport entre le nombre d'électrons récupérés (un courant) au nombre de photons incidents (une puissance). La température de fonctionnement dépend de la longueur d'onde d'absorption. Ainsi pour un QWIP possédant un pic de détection à 9 micronmètre (courbe bleue, figure 2), la température de fonctionnement est de $77 \mathrm{~K}$ (température de l'azote liquide).

La couche active des QWIPs est une hétérostructure à base de semiconducteurs. La structure est obtenue en réalisant une succession périodique de couches d'AlGaAs et d'AsGa. La conception de la structure (épaisseur, composition des différentes couches, dopage des couches ...) permet de choisir la bande de détection (figure 2). Par exemple, les couches AsGa pour une bande de détection centrée à 9 micronmètre ont une épaisseur de $5 \mathrm{~nm}$ soit une vingtaine de couches atomiques. On réalise une couche active par une technique d'épitaxie telle que l'épitaxie par jets moléculaires. On dépose ainsi des atomes, couche atomique par couche atomique sur des galettes de substrats mono-cristallins. Cette technique permet de maîtriser les interfaces à la mono-couche atomique près, elle garantit en outre une excellente uniformité de composition sur de grandes surfaces et une grande pureté du matériau synthétisé. Le détecteur QWIP n'absorbe pas le rayonnement arrivant à incidence normale. Il faut alors prévoir une structure de couplage optique sur chaque élément détecteur (ou pixel), un réseau de diffraction travaillant en réflexion constitue cette structure de couplage (figure 3). 


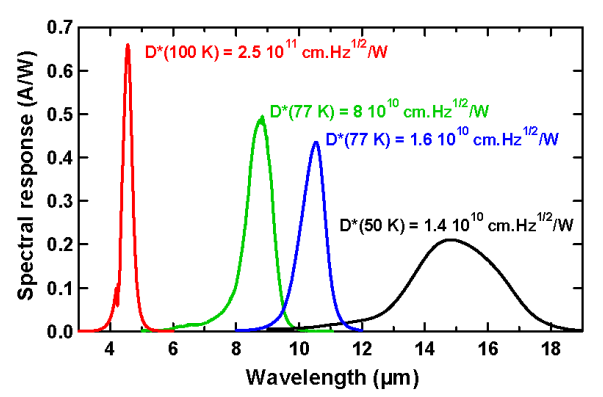

Figure 2. Absorptions résonantes des détecteurs QWIP.

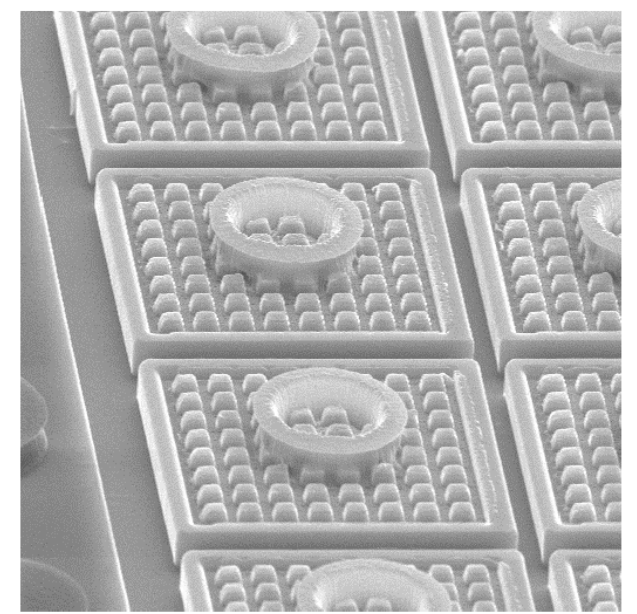

Figure 3. Motifs des matrices des détecteurs QWIP.

Le détecteur QWIP doit être refroidi car les transitions électroniques permettant son fonctionnement peuvent être excitées par les phonons qui correspondent aux vibrations du réseau cristallin. Pour réduire ces vibrations, il faut refroidir le détecteur, la température de fonctionnement dépend de la bande de fréquence de fonctionnement.. Dès que la couche active est obtenue, il est alors possible de réaliser des matrices de détecteurs par des procédés technologiques de gravure (photolithographie à l'aide de résines photosensibles, gravure sèche, gravure plasma ...). On grave la structure de couplage optique, les pixels et on réalise ensuite les contacts électriques. On peut obtenir des matrices au format dit TV (640x512 pixels). Les pixels ont des dimensions allant de 15 à 25 micronmètre. La matrice de détecteurs ainsi obtenue est connectée à l'aide de billes d'indium à une matrice similaire composée de circuits de lecture en silicium qui polarisent le détecteur et recueillent le signal, cette opération d'assemblage s'appelle l'hybridation. L'hybride obtenu est monté dans un cryostat couplé à une machine à froid, l'ensemble forme le bloc détecteur (Figure 4). L'ensemble pèse 400 grammes et mesure $15 \mathrm{~cm}$. Le bloc détecteur est ensuite intégré dans une caméra comprenant l'optique, l'électronique de lecture, le traitement d'image (Figure 5). Le contraste thermique pouvant être décelable est de l'ordre d'une dizaine de mK. Ce qui donne des sensibilités compatibles des applications de thermographie médicale. 


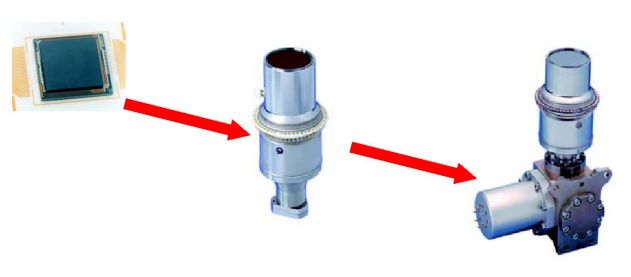

Figure 4. Intégration du bloc détecteur.

\section{IMAGERIE ACTIVE ET IMAGERIE LASER}

Dans le chapitre précédent, nous avons décrit les techniques d'imagerie optique ou infrarouge passive et leurs technologies associées. Dans ce chapitre, nous allons nous intéresser aux techniques d'imagerie active. Ces techniques comprennent une source permettant l'illumination de l'échantillon biologique à étudier, un système optique, un système de détection permettant la réception du signal transmis ou réfléchi par l'échantillon, un système de traitement de l'information qui va fournir l'image désirée. La source peut être une source possédant une extension spectrale ou une source laser. Le système de détection peut être un mono-détecteur ou une matrice de détecteurs. L'imagerie active proche infrarouge est une méthode d'évaluation non invasive, elle permet en outre le contrôle de la source d'illumination, de la longueur d'onde, de la polarisation de l'onde incidente et de la durée de l'impulsion. Les principales applications des techniques d'imagerie optique active en médecine sont : - le cancer du sein pour la détection des tumeurs, - le cancer de la peau et notamment l'imagerie du derme et de l'épiderme pour améliorer le contraste entre un mélanome bénin et malin, - l'imagerie fonctionnelle des extrémités afin étudier les maladies musculaires comme les maladies vasculaires, - l'imagerie du cerveau du nourrisson.

Nous pouvons diviser ces techniques en deux grandes catégories : l'imagerie en mode réflexion et l'imagerie en mode diffusion. La difficulté majeure pour créer un système d'imagerie utilisant la lumière proche infrarouge provient de la propriété de diffusion des tissus, ceci donne une image " floue " et masque les structures les plus profondes. Il est alors indispensable de séparer les photons qui vont être multidiffusés et les photons ne subissant qu'un nombre très faible n'interactions et " gardant la mémoire " de leur interaction avec le tissu à étudier. Ces photons constituent alors l'information nécessaire pour construire l'imagerie optique.

Nous allons rapidement décrire l'imagerie en mode diffusion. Afin d'obtenir un rapport signal à bruit suffisant dans le cadre de milieu diffusant, il est nécessaire d'éliminer les photons multidiffusés et de garder les photons porteurs de l'information utile pour construire l'image du milieu diffusant, ce que nous pouvons appeler les photons balistiques. Deux possibilités nous sont offertes, la sélection des photons à la réception ou la sélection des photons à l'émission. Une troisième technique sera présentée, il s'agit des méthodes de reconstruction d'images.

Nous allons maintenant étudier les techniques de sélection à la réception. Différentes approches sont utilisées :

- sélection spatiale : les photons dits balistiques se propagent en ligne droite, il suffit de filtrer spatialement les photons multidiffusés se propageant en dehors de l'axe de propagation des photons balistiques. En transmission, nous pouvons ainsi utiliser un filtre spatial placé dans le plan de Fourier du système optique. Ces techniques sont utilisées pour l'imagerie plein champ. Mais on peut adopter une stratégie différente comme l'imagerie confocale. Cette méthode nécessite un balayage de l'échantillon. Elle peut être utilisée en diffusion et en imagerie de la fluorescence, - sélection temporelle : les photons balistiques se propagent en ligne droite, par conséquent la durée des trajets suivis par ces photons est plus courte que celles des photons multidiffusés ou serpentiles. La notion de photons serpentiles reste empirique, en effet nous allons appeler photons serpentiles les photons peu diffusés dont le trajet reste autour du trajet balistique. Ces photons apportent une information intéressante 


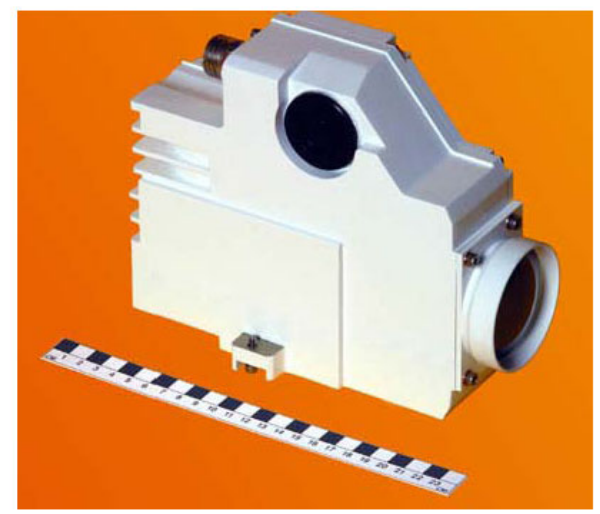

Figure 5. Caméra Catherine QWIP de Thales Optronique, Poids : $2,5 \mathrm{~kg}$, Volume :2 dm3.

sur le milieu diffusant. L'idée sous-jacente à la sélection temporelle consiste à envoyer une impulsion lumineuse très brève dans le tissu et à ne récupérer que les photons les plus rapides. La difficulté principale de cette technique est la réalisation technologique d'une porte temporelle suffisamment rapide séparant la détection des photons balistiques de la détection des photons multidiffusés. L'optronique permet de concevoir ce type de porte temporelle, les photomultiplicateurs possèdent ainsi des temps de réponse de l'ordre de plusieurs dizaines de picosecondes. D'autres types de détecteurs sont aussi utilisés pour mesurer une intensité, une caméra à balayage de fente ou un système de comptage de photons corrélés dans le temps. Les inconvénients majeurs de la caméra à balayage de fente sont sa non-linéarité et sa largeur de sa fenêtre temporelle limitée à quelques nanosecondes. Les phénomènes optiques non linéaires, génération de la seconde harmonique, amplification paramétrique optique, effet Kerr optique (une résolution submillimétrique peut être obtenue), effet Raman stimulé peuvent fournir des portes temporelles rapides.

La sélection à l'émission consiste à réaliser une source liée aux propriétés optiques du milieu diffusant. Cette approche permet de repousser la frontière est entre photons diffusés et serpentiles, tout en gardant une bonne résolution spatiale, la résolution spatiale et la profondeur de pénétration étant liées à la réalisation de la source focalisée en profondeur. Il suffit alors d'exploiter un certain nombre de phénomènes non linéaires pour induire l'émission de lumière par le tissu à une autre longueur d'onde que celle de la source d'excitation. La suppression de la lumière excitatrice est alors simplement réalisée par filtrage.

Nous avons vu que, dans de nombreux cas, l'imagerie directe ne permet pas une profondeur de pénétration de l'onde optique importante. Ainsi pour pouvoir explorer des tissus très épais, il est nécessaire de détecter tout le signal optique multi-diffusé. L'idée de base est simple, pour une position émetteur-récepteur donnée, un modèle de propagation lumineuse permet de simuler l'expérience réalisée (c'est que nous avons coutume d'appeler le problème direct). La reconstruction de l'image consiste alors à résoudre un problème inverse. Résoudre le problème inverse consiste à optimiser un critère représentant l'écart entre l'expérience et la modélisation. Cette méthode est connue sous le nom de Tomographie Optique Diffuse (TOD). Nous pouvons alors définir le principe de la tomographie, cette technique utilise une géométrie source-détecteur afin que l'onde électromagnétique traverse des régions situées en profondeur, ce qui produit une image des structures internes. L'ensemble des positions émetteur récepteur va permettre de fournir une image bi-dimensionnelle voire tri-dimensionnelle. La profondeur d'exploration est ici uniquement limitée par les performances du système de détection. La résolution spatiale dépend des performances du système expérimental, mais elle est liée à la modélisation et au processus mathématique d'inversion.

Nous définissons alors trois types de Tomographie Optique Diffuse suivant les données recueillies : - TOD continue : ces systèmes utilisent une source continue et mesurent l'intensité totale à la surface du tissu. Ce 
système offre de bonnes performances en terme de stabilité et de rapport signal à bruit. Leur désavantage est lié au fait qu'ils ne recueillent que peu d'informations et ceci impose alors la multiplication du nombre de mesures. - TOD fréquentielle : les systèmes fréquentiels utilisent des sources modulées à des fréquences radios. Ces systèmes mesurent l'intensité totale et le déphasage entre les signaux incident et détecté et la variation de l'indice de modulation du signal. De performances équivalentes et de coût voisin aux systèmes continus, ils permettent de recueillir plus d'informations que ceux-ci. - TOD résolue en temps : les systèmes résolus en temps utilisent des sources impulsionnelles et mesurent le profil temporel de l'intensité lumineuse. Dans ces dispositifs, des impulsions lumineuses ultracourtes (picoseconde ou femtoseconde) éclairent la surface du tissu biologique. L'intensité transmise par le tissu est détectée en fonction du temps d'arrivée des photons avec une résolution de l'ordre de la picoseconde. Ce qui consiste à mesurer les temps de vol des photons à travers l'objet étudié en plusieurs points de sortie. L'utilisation d'une onde impulsionnelle et monochromatique nécessite l'emploi d'un laser, solide, à colorant ou semi-conducteur. Divers types d'instrumentation optoélectronique sont utilisés pour la détection, notamment les systèmes de comptage de photons. Dans ce cas, l'intensité transmise correspond à la réponse impulsionnelle du tissu et est fonction des propriétés optiques du tissu traversé. Le temps de propagation d'un photon de la source au détecteur est déterminé par la longueur du chemin suivi. Plus le milieu est diffusant, plus le chemin suivi est statistiquement long et plus le temps d'arrivée moyen des photons sera élevé. Au contraire, plus le milieu est absorbant, plus le nombre de photons émergeant du tissu biologique après un temps long sera faible à cause de la probabilité d'absorption. Le temps d'arrivée moyen des photons sera donc diminué. La répartition du temps d'arrivée des photons est plus ou moins large suivant les propriétés physiques des milieux traversés.

La méthode la plus simple pour reconstruire une image à partir de mesures de tomographie optique est connue sous le nom de rétro-projection. Les mesures des profils temporels pour un ensemble de positions émetteurdétecteur, appelées projections, doivent permettre de reconstruire les images d'absorption et de diffusion de la structure biologique hétérogène. La tomographie est une cartographie pouvant être tridimensionnelle des propriétés optiques des régions situées à l'intérieur du volume examiné.

Nous allons maintenant nous intéresser aux techniques d'imagerie en mode réflexion. Ces techniques en réflexion présente l'intérêt de la mesure in vivo. Nous allons ainsi définir deux types d'imageries : o la topographie qui peut être caractérisée comme une cartographie des propriétés projetées sur la surface externe de l'organe étudié, o la tomographie qui est alors caractérisée par une cartographie tridimensionnelle des propriétés optiques des régions situées à l'intérieur du volume examiné. Il faut alors remarquer que les coupes tomographiques deviennent des images bi-dimensionnelles en profondeur.

Les techniques de sélection précédemment discutées s'appliquent de la même façon en imagerie en réflexion. Mais il faut remarquer que si nous abandonnons les techniques classiques d'imagerie plein champ, nous allons définir alors l'imagerie de microscopie confocale correspondant à un balayage de l'échantillon. Nous avons ainsi en réflexion un filtrage spatial, et une image en trois dimensions. L'objectif du microscope permet de focaliser la source et de faire l'image du point de focalisation sur le détecteur. En plaçant un diaphragme devant le détecteur, il est alors possible de filtrer la lumière multidiffusée et la lumière rétrodiffusée depuis les plans autres que celui de focalisation. Les résolutions commerciales sont de l'ordre du micron. En additionnant cette technique à une technique d'imagerie fluorescente donc de sélection à l'émission, il est possible d'augmenter fortement la résolution spatiale. Ainsi pour l'absorption multiphotonique, la source excite l'émission d'agents fluorescents par l'absorption de plus d'un photon, en général deux ou trois. Un avantage important de cette technique par rapport à la microscopie de fluorescence traditionnelle réside dans le fait l'excitation à n photons se fait à une fréquence $\mathrm{n}$ fois plus petite que l'excitation monophotonique. Ainsi il est possible d'exciter les fluorophores connus dans le visible ou les UV avec des longueurs d'onde infrarouges. Ceci permet d'accroître naturellement la pénétration de l'onde dans le tissu biologique et de réduire la phototoxicité induite.

Une autre technique importante d'imagerie en réflexion est liée à la sélection par cohérence temporelle. Nous savons que deux ondes lumineuses ne peuvent interférer que s'il existe une relation de cohérence temporelle entre elles. La Tomographie Optique Cohérente ou OCT (Optical Coherence Tomography) utilise le principe 


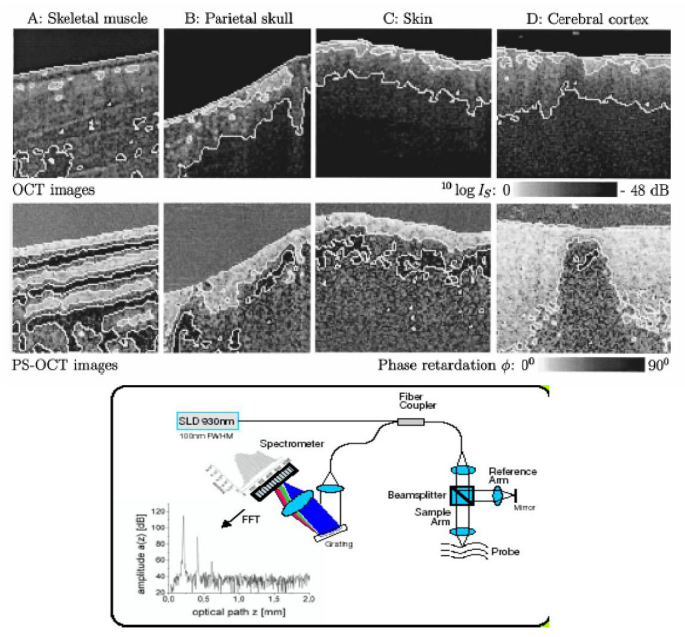

Figure 6. Images OCT par De Boer et al. (University of California, Irvine), les images PSOCT exploitent la polarisation de l'onde afin d'améliorer les contrastes.

de l'interférométrie à faible longueur de cohérence, cette technique a été initialement proposée par J.G. Fujimoto. Elle consiste à balayer transversalement l'échantillon observé en réflexion en utilisant une architecture de microscopie confocale. La profondeur est définie par une porte temporelle utilisant le phénomène d'interférence entre le signal d'intérêt et une référence. La durée d'ouverture de la porte temporelle et en conséquence la résolution en profondeur est définie par la durée de cohérence de la source. La résolution atteignable in vivo est de l'ordre du micron et la profondeur de l'ordre de plusieurs millimètres (Figure 6). On définit habituellement deux types d'OCT. Le premier type correspond à l'OCT dans le domaine temporel, on effectue alors une modulation temporelle du chemin optique de la voie de référence, ce qui permet un balayage de l'échantillon en profondeur. Le deuxième type d'OCT est défini dans le domaine de Fourier, il est caractérisé par une détection spectrale ce qui permet d'éviter la modulation du chemin optique de référence. Ces méthodes nécessitent le balayage transversal de l'échantillon afin d'obtenir une image tomographique. Certaines méthodes dites OCT plein champ vont s'affranchir de ce balayage et diminuer en conséquence le temps d'acquisition de l'image. Les techniques de polarisation peuvent être alors utilisées pour améliorer le contraste de l'image (Figure 6). L'OCT est bien adapté pour les études dermatologiques (détection précoce des cancers de la peau) et l'ophtalmologie.

Nous allons étudier l'imagerie topographique bi-dimensionnelle en réflexion. Cette technique peut être multispectrale et polarimétrique, c'est-à-dire utiliser plusieurs longueurs d'onde ou bandes et l'analyse polarimétrique du signal rétroréfléchi. Nous avons ainsi une technique non invasive, permettant le contrôle de la source d'illumination, de la longueur d'onde, de la polarisation et de la durée de l'impulsion de l'onde incidente. Elle peut être utilisée pour la quantification des désordres pigmentaires et de la détection de mélanomes par exemple. Le champ image est important, la résolution spatiale peut atteindre le micron. L'imagerie topographique en réflexion peut être : - une imagerie directe polarimétrique permettant d'obtenir une image de surface (image de la peau par exemple) directement liée aux aspérités et indices des premières couches de surfaces, - une image directe polarimétrique et multispectrale permettant d'obtenir une image spectroscopique en profondeur grâce aux différents choix de longueurs d'onde et de propriétés électromagnétiques des différents constituants.

L'utilisation d'impulsions très brèves (lasers femtoseconde) et de plusieurs plans images peut permettre alors une reconstruction tomographique de la structure explorée, nous pouvons obtenir ainsi une image en volume.

La figure 7 décrit un système d'imagerie polarimétrique et multispectrale en réflexion. 


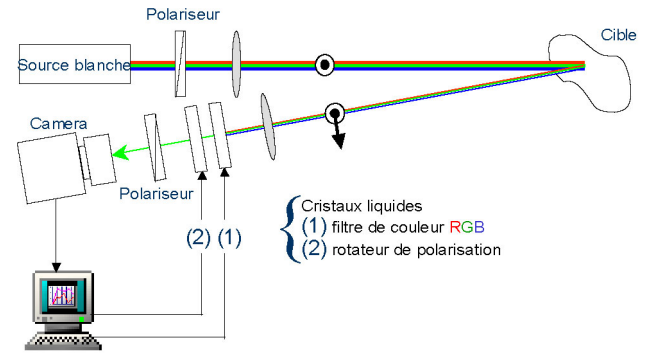

FiguRE 7. Système d'imagerie direct polarimétrique et multispectrale en réflexion, ce système est décrit avec une source de lumière blanche, un laser accordable peut être utilisé avec ce type de système.

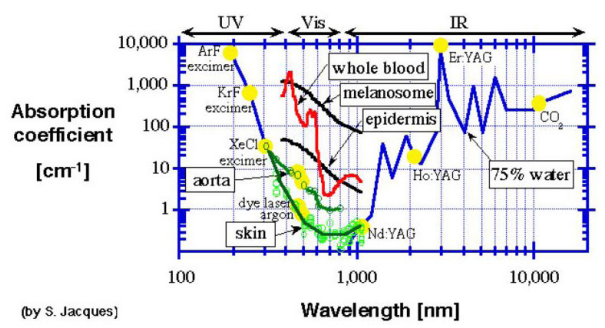

Figure 8. Coefficient d'absorption des différents éléments d'un tissu biologique, les points jaunes représentent les différentes longueurs d'onde laser. Nous voyons ici l'intérêt d'une analyse multispectrale.

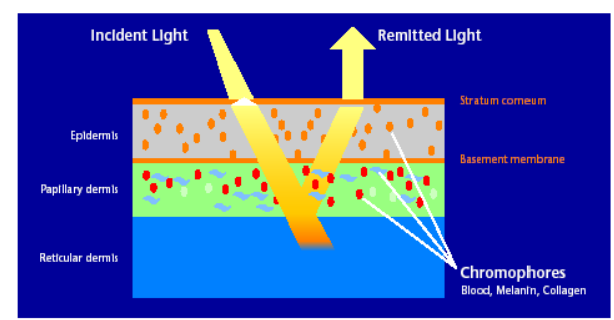

Figure 9. Modèle de la peau.

L'imagerie multispectrale nous permet d'obtenir une information sur les composants situés en profondeur, la figure 8 détaille ainsi les coefficients d'absorption des différents composants d'un tissu biologique. Ces composants peuvent être ainsi discriminés par leur comportement électromagnétique dépendant de la longueur d'onde d'illumination. La figure 8 montre ainsi une image plein champ multispectrale permettant l'identification de différents éléments de la peau qui peuvent être modélisés comme l'indique la figure 9. 

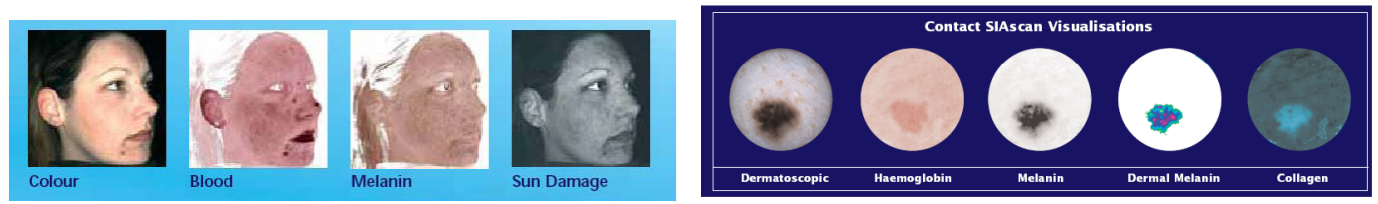

Figure 10. (a) images plein champ par le système SIAscopy d'Astron Clinica. Ce système multispectral permet une identification des différents constituants de la peau grâce à une comparaison avec une base de données calculées. (b) images petit champ " in vivo " et identification des différents constituants.

Pour illustrer cette technique d'imagerie en réflexion, nous allons nous concentrer sur l'imagerie de la peau. La connaissance des désordres pigmentaires, l'identification précoce des structures malignes est un point particulièrement sensible de l'analyse optique de la peau. Le système d'imagerie multispectrale par réflexion permet de déterminer la nature, la position des différents composants de la peau en particulier les quantités d'hémoglobine, de mélanine et de collagène dans la structure de la peau. L'information peut être présentée sous forme de cartes bi-dimensionnelles ou topographique. Le système dont les images sont présentées sur la figure 10 utilise une lumière blanche et un ensemble de filtres permettant d'obtenir différentes bandes spectrales. La lumière blanche filtrée peut être remplacée par plusieurs sources monochromatiques (diodes laser, diodes électroluminescentes). L'identification sera d'autant plus fiable qu'elle contient un algorithme permettant une comparaison de la mesure à une base de données. Cette technique de comparaison nécessite des algorithmes d'identification. Le système comprend donc une base de données calculées ou mesurées. Dans le cas d'une base de données calculées, il est nécessaire de mettre en place un modèle mathématique de l'interaction de la lumière avec la structure de la peau et des différents chromophores, de connaître leurs propriétés spectrales. Ceci permet d'analyser la peau avec des techniques de spectro-photométrie large bande. Cette technique donne une cartographie bidimensionnelle de l'absorption et de la diffusion à partir de données mesurées sur la structure hétérogène de la peau. Un point important dans ces techniques d'imagerie en réflexion est de traiter la première diffusion sur la face externe de la peau afin d'augmenter le rapport signal à bruit de l'interaction lumière/structure interne. Les techniques d'analyse de polarisation peuvent être utilisées en mesure, en effet l'interaction avec la surface est faiblement dépolarisante dans le plan d'incidence alors que l'interaction avec le volume est fortement dépolarisante. L'ajout de la polarisation dans un système multispectral permet de modifier le contraste de l'information optique enregistrée. La polarimétrie de Mueller en réflexion a démontré ses capacités à contraster des structures biologiques différentes, mais il est nécessaire de progresser sur l'interprétation physiologique des matrices de Mueller calculées. L'apport d'un modèle numérique est essentiel pour passer d'une imagerie de contraste à une imagerie interprétative de désordres structurels des tissus explorés. Cette dernière repose sur des caméras permettant, avec une grande précision, l'estimation de l'état de polarisation de la lumière rétrodiffusée par la peau examinée. Une telle caméra doit combiner des détecteurs matriciels à grande dynamique (sensibles dans le visible et le proche infrarouge) avec un générateur d'états de polarisation programmable, rapide et large bande de manière à permettre le fonctionnement multispectral. Les images polarimétriques sont obtenues en utilisant les matrices de Mueller et en extrayant des indicateurs comme par exemple le degré de polarisation qui est défini (pour sa définition la plus simple) comme le rapport de la différence des intensités en polarisation verticale et horizontale sur la somme de ces deux intensités. Ce degré de polarisation sera égal à 1 si l'échantillon ne dépolarise pas la lumière incidence et à 0 dans le cas contraire. Il est alors possible de concevoir un système donnant le degré de polarisation sur chaque pixel du détecteur. Un exemple d'une image en degré de polarisation est donné sur la figure 11. 


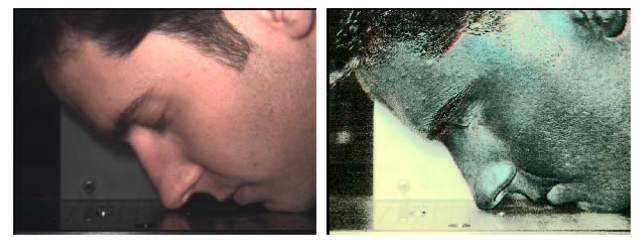

Figure 11. Comparaison entre une image couleur et une image plein champ en degré de polarisation.

\section{MODELISATION}

\subsection{Modèle optico-physiologique de la peau et des tissus}

Les processus d'absorption sont liés à l'énergie du photon, par conséquent l'absorption d'un chromophore varie avec la longueur d'onde. Le spectre d'absorption d'un milieu complexe est la somme des spectres de ses différents constituants. Nous allons définir les principaux chromophores.

L'hémoglobine L'absorbant principal des tissus est l'hémoglobine du sang (dans son état oxydé ou non). Elle est responsable du transport de l'oxygène par les hématies dans le sang, où elle est présente sous deux formes majoritaires, l'oxy-hémoglobine et la déoxy-hémoglobine. La concentration d'hémoglobine dans les hématies est d'environ $350 \mathrm{~g} / \mathrm{L}$. En considérant un taux hématocrite moyen de 0,45 on obtient une concentration d'hémoglobine dans le sang d'environ $160 \mathrm{~g} / \mathrm{L}$. Les deux spectres d'absorption sont proches dans le visible mais très différents dans la bande proche infrarouge.

Le cytochrome c oxydase

C'est l'enzyme terminale dans le cycle respiratoire de la cellule. Son absorption est du même ordre de grandeur que celle de l'hémoglobine dans la bande proche infrarouge mais sa concentration dans les tissus lui est inférieure d'au moins un ordre de grandeur.

Les lipides

Leur absorption est du même ordre de grandeur que celle de l'eau dans la bande infrarouge.

La mélanine

Elle assure un rôle protecteur dans l'épiderme et dans les cheveux. Elle absorbe préférentiellement dans l'UV et le visible.

D'autres chromophores tel l'ADN ou les protéines jouent un rôle dans l'absorption des tissus biologiques.

La peau

En plus des chromophores habituels, l'absorption de la peau est aussi due à la présence de mélanine dans l'épiderme en quantité fortement variable suivant le type de peau. Nous avons vu que la mélanine absorbe fortement dans l'UV et le visible. La diffusion dans la peau est en partie due aux fibres de collagène. Au niveau macroscopique, leur structure fibrée se traduit par un régime de diffusion de Mie, fortement anisotropique. Toutefois, des stries microscopiques ajoutent un régime de diffusion de Rayleigh de nature isotropique.

\subsection{Modèles de transport et de diffusion de la lumière dans les tissus et la peau}

Ces modèles sont indispensables à l'interprétation des images complexes polarimétrique, multispectrales, bi-dimensionnelles voire tri-dimensionnelles et de leur lien avec des désordres cutanés pouvant être reliés à la détection précoce des cancers. Ils relient ainsi l'ensemble des variations de paramètres physiques des tissus associées (par exemple : absorption, densité, structure, nature, forme des hétérogénéités, altérations structurelles ou morphologiques de la peau) aux paramètres de la lumière mesurés en réflexion ou en diffusion (intensité, polarisation,...). La structure fine, l'hétérogénéité des couches, leur empilement rendent cette modélisation de l'interaction onde-tissu biologique particulièrement difficile. Cependant elle est indispensable à l'interprétation des images et des mesures, elle intervient dans la mise en place des problèmes inverses servant à l'identification des désordres présents dans les tissus mesurés. 


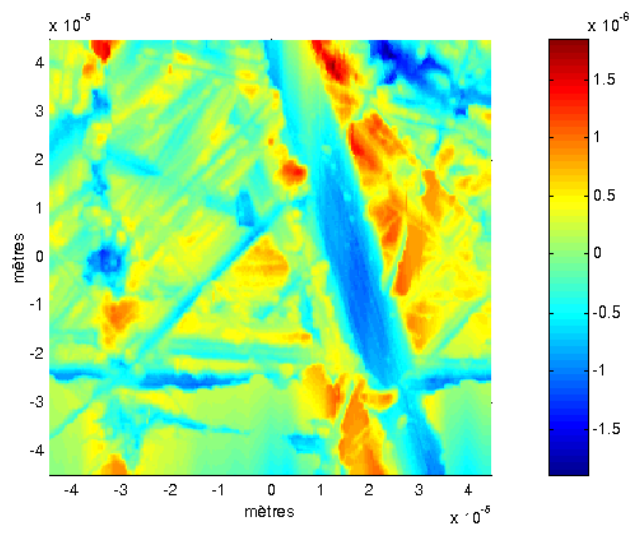

Figure 12. Mesure (Atomic Force Microscope) et représentation des aspérités d'une surface rugueuse aléatoire, ce type de surface se caractérise par une densité de probabilité des hauteurs gaussienne et une fonction d'autocorrélation non-gaussienne. L'aspect non-gaussien de la fonction d'auto-corrélation prend en compte les structures en strie de l'interface.

Modèles de diffusion de la lumière par les structures rugueuses composant les interfaces d'un tissu

Le premier problème à traiter est la modélisation de la diffusion électromagnétique par l'interface externe du tissu considéré. Dans le cas de la peau, cela correspond à la diffusion par la structure externe de celle-ci. La première partie du modèle de diffusion électromagnétique consiste à caractériser cette surface par une densité de probabilité des hauteurs et une fonction de corrélation représentant les deux échelles du processus aléatoire. En effet, la structure externe de la peau est une surface rugueuse aléatoire. Le modèle statistique permet de décrire la surface tridimensionnelle de la peau grâce à une fonction bidimensionnelle des probabilités des hauteurs et une fonction bidimensionnelle de corrélation. Ces deux fonctions décrivent les micro-structurations et l'organisation de celles-ci dans un plan. La mesure de l'état de surface de la peau permet de construire le modèle statistique. La modélisation de la fonction de corrélation est un point particulièrement important pour le calcul de la diffusion électromagnétique. Ce modèle décrira statistiquement les micro-aspérités tridimensionnelles de la peau comme nous pouvons l'observer sur la figure 12. Il faut ensuite développer un modèle spécifique de diffusion d'une onde lumineuse par une surface rugueuse aléatoire décrivant la peau externe ou l'interface du tissu considéré. Les modèles sont des extensions des théories de diffusion comprenant les approximations de faible pente (ou SmallSlope Approximation, SSA) dans le cas de surfaces non gaussiennes, c'est-à-dire comprenant dans ce cas une fonction d'auto-corrélation non Gaussienne. Ce modèle permettra de calculer la section efficace de l'ensemble des états de polarisations de l'onde incidente appartenant au spectre visible ou proche infrarouge. Nous préférons ce type de modèle perturbatif aux modèles d'équations intégrales, d'éléments finis ou de différences finies afin de traiter des cas réalistes de surfaces tridimensionnelles complexes. Les méthodes d'approximation faible pente donnent des résultats excellents sur des surfaces tri-dimensionnelles complexes (figure 13). Les modèles approchés de Kirchhoff peuvent être utilisés mais leur domaine de validité est beaucoup plus restreint que les modèles d'approximation de faible pente.

Modèles de diffusion et de transport de la lumière par les volumes aléatoires limités par des surfaces rugueuses aléatoires

Le second point de la modélisation concerne le volume interne de la peau ou des tissus. Ils sont modélisés par un volume aléatoire comprenant des distributions de microstructures disposées aléatoirement dans le volume. D'un point de vue macroscopique, on considère généralement qu'un milieu réel est constitué de populations de différents chromophores réparties localement de façon uniforme. Chaque chromophore est assimilé à une sphère d'une taille proportionnelle à son pouvoir d'absorption. L'analyse de la répartition et de la concentration des chromophores, de leurs différents indices permet de conclure à une modélisation des couches internes de la peau 

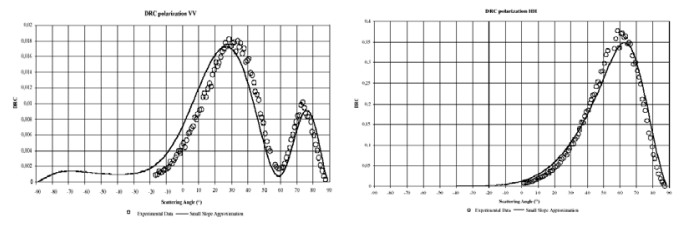

Figure 13. Comparaison de l'intensité diffusée mesurée et calculée (Small-Slope Approximation) [G. Berginc in Progress in Electromagnetic Research (J.A. Kong ed.), EMW Publishing, $(2003)]$

par des structures diélectriques pouvant être décrites par des indices effectifs aux longueurs d'ondes examinées ou par des volumes aléatoires comprenant des inclusions. Ce modèle de l'indice des couches internes de la peau constitue un modèle optico-physiologique complet. Les modèles d'indices effectifs permettent une simplification du modèle d'interaction électromagnétique. Le modèle de diffusion volumique de la peau concerne l'élaboration de l'équation de transport de la lumière polarisée à l'intérieur des tissus biologiques et la diffusion de celle-ci à travers les différentes interfaces présentes à l'intérieur de la structure biologique. Deux méthodes de modélisation sont couramment utilisées pour simuler la diffusion d'une onde lumineuse à l'intérieur des tissus : - les méthodes de Monte Carlo, elles consistent à faire interagir un nombre important de photons avec les chromophores. L'interaction est décrite comme une diffusion ou une absorption. Cette technique reste très coûteuse en temps de calcul et très difficilement applicable à une structure complexe comme les tissus biologiques sans y apporter des simplifications, le couplage interface rugueuse aléatoire et volume aléatoire devient très complexe à traiter, - la résolution numérique de l'équation de propagation de la lumière qui se réduit à l'équation de diffusion dans un milieu fortement diffusant. La méthode des éléments finis est alors employée pour résoudre l'équation de diffusion. La simplification couramment utilisée est de faire abstraction des phénomènes interférométriques et diffractifs, liés à la nature ondulatoire de la lumière. On ne considère que la propagation de l'énergie lumineuse. Cette approche repose sur la théorie du transfert radiatif phénoménologique qui est fondée sur l'absorption et la diffusion de l'onde lumineuse par les constituants de la structure. Cette approche, bien que gourmande en temps de calcul, ne permet pas de prendre en compte l'ensemble des phénomènes physiques notamment les couplages interfaces-volumes. Le calcul dans les cas d'objets tri-dimensionnel devient hors de portée.

Un troisième modèle présente un avantage par rapport aux modèles classiques, il tient compte de l'ensemble des états de polarisation de la lumière et du couplage avec les interfaces rugueuses qui agit directement sur la dépolarisation de l'onde lumineuse. La diffusion de la lumière polarisée par un système tridimensionnel complexe peut être modélisée grâce à cette technique. La procédure utilisée permet d'écrire les équations de Maxwell sous une forme intégrale avec l'aide des fonctions de Green et prendre en compte de nouvelles contributions comme la diffusion de la lumière par les interfaces rugueuses, les effets de corrélations entre les diffuseurs et les phénomènes de " backscattering enhancement " liés aux interférences constructives. Le milieu aléatoire est composée de particules dont la répartition est décrite par une p-d f (pair-distribution function) L'intensité diffusée est donnée par l'équation de Bethe-Salpeter. Nous allons aborder comme exemple le problème des nanomateriaux inclus dans une couche rugueuse biologique. La dissémination de ces nanocomposants dans le corps humain est très mal connue. Il est hors de propos de faire un calcul complètement réaliste, tenant compte de l'ensemble des paramètres biologiques. Le but est de mettre en évidence les différents mécanismes d'interaction. Nous prenons une structure de peau qui est considérée comme une couche diélectrique bornée par une surface rugueuse aléatoire. Dans ce volume tri-dimensionnelle sont introduites des nanobilles de noir de carbone. Ce modèle traite d'une façon assez réaliste de l'introduction de polluants dans l'organisme. La structure de la peau repose sur un milieu semi-infini constitué par le muscle. La structure externe de la peau est considérée comme une surface rugueuse aléatoire décrite par une densité de probabilité et une fonction d'autocorrélation gaussiennes. Les propriétés électromagnétiques de la peau sont modélisées par un indice effectif. 

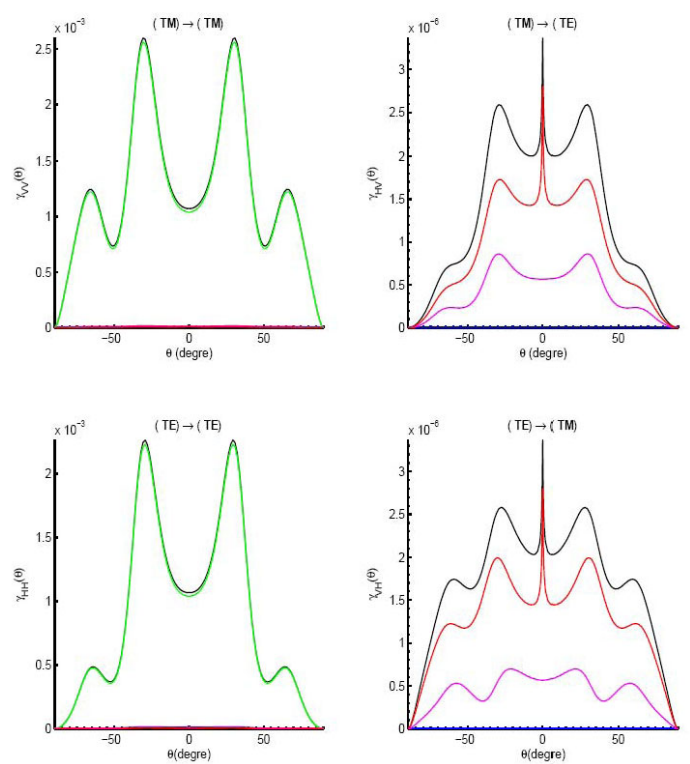

Figure 14. Processus de diffusion simple par la surface (traits verts), processus de diffusion simple par le volume (traits bleus). Contribution surface rugueuse et un diffuseur (traits magenta). Contributions du second ordre (traits rouges). Somme totale des contributions (traits noirs).

Nous prenons comme longueur d'onde laser incidente $=650 \mathrm{~nm}$. La fraction volumique des nanobilles est de l'ordre de 1/10000. Nous considérons deux types de nanobilles de rayons différents égaux à $1 / 40$ et $1 / 30$ de la longueur d'onde. La structure globale est tri-dimensionnelle, le calcul est effectué dans le plan d'incidence de l'onde laser éclairant normalement la surface. L'épaisseur de la couche de peau est inférieure à la largeur d'impulsion de l'onde laser. Sur la figure 14, nous donnons les sections efficaces en réflexion de l'ensemble des contributions qui sont décrites de la façon suivante: - processus d'interaction avec la surface rugueuse supérieure, - processus de diffusion simple dans le volume, - processus de couplage d'un diffuseur avec la surface, - processus du second ordre, ce type d'interaction va comprendre des diffusions doubles et triples nanobilles et surfaces rugueuses.

L'exemple ainsi calculé permet de démontrer l'importance de la diffusion par la surface rugueuse aléatoire dans les polarisations principales et dans le plan d'incidence. Ce processus de diffusion porte l'ensemble de la contribution à la section efficace totale, il serait alors impossible de mesurer l'influence des polluants en polarisation principale sans retirer cette contribution. En effet, l'ensemble permittivité de la couche et des nanobilles conduit à une partie imaginaire de la permittivité effective assez élevée, ce qui se traduit par des intensités diffusées importantes. Pour les polarisations croisées, on met en évidence les effets du premier et second ordre de la diffusion. Les contributions du volume aléatoires sont dans ce cas les effets majeurs. Il est intéressant de constater que les effets des processus de diffusion simple dans le volume sont négligeables par rapport aux effets des processus du second ordre. Nous avons ainsi démontré l'intérêt d'un modèle permettant l'exploitation des interactions surface/volume et du calcul sur l'ensemble des polarisations. La possibilité d'analyser les différents contributeurs suivant les ordres du développement perturbatif de la solution à l'équation générale de transport de la lumière est un point particulièrement intéressant notamment pour la spécification d'un système de détection d'anomalies dans des tissus biologiques. 


\subsection{Algorithme de reconstruction}

Le problème direct est particulièrement important en vue de la reconstruction tomographique. La connaissance du problème direct est alors exploitée pour l'identification de désordres cutanés par exemple et la reconstruction tomographique. L'algorithmie de reconstruction est un point fondamental de la spécification d'un appareillage de tomographie. Mais la lumière qui traverse un tissu biologique subit très vite le phénomène de diffusion, il est alors difficile de déduire les propriétés optiques internes d'absorption par simple rétroprojection, comme c'est le cas pour l'imagerie de rayons X. De plus, tout comme l'absorption, la diffusion atténue la lumière. Ainsi, en mesurant uniquement l'intensité lumineuse issue du tissu pour une fréquence, il est difficile de séparer les effets dus à l'absorption, de ceux dus à la diffusion. Les principes mathématiques de reconstruction ont été développés dès 1917 par Radon, bien longtemps avant les premiers prototypes d'appareils d'imagerie tomographique. Beaucoup de travaux mathématiques et numériques récents dans le domaine des problèmes d'inversion ont été consacrés à la détermination des propriétés électromagnétiques de structures éclairées par une onde lumineuse. De nouveaux algorithmes (MUltiple SIgnal Classification, MUSIC) sont apparus et permettent d'envisager des techniques d'identification plus performantes. Il est particulièrement important de constater que le problème direct et le processus de reconstruction sont intimement liés.

\section{CONCLUSIONS}

Grâce au développement des dispositifs d'imagerie optique conventionnelle (imagerie thermique) ou non conventionnelle (imagerie OCT, microscopie confocale, imagerie tomographique diffuse, imagerie active polarimétrique et multispectrale ...), nous disposerons d'images "complexes", combinant des contrastes d'une très grande richesse avec une grande sensibilité, et dont l'interprétation sera rendue possible par la disponibilité de la modélisation des interactions ondes-tissus et d'algorithmes de reconstruction stables et performants. Cet ensemble d'outils technologiques et mathématiques permettra d'analyser et d'identifier les pathologies grâce aux mesures des variations des différents paramètres physiques des tissus biologiques. 